\title{
Diagnosis of ventriculo-arterial discordance (transposition of the great arteries) by contrast echocardiography
}

\author{
CARLOS MORTERA, STEWART HUNTER, AND MICHAEL TYNAN \\ From Department of Cardiology, Newcastle General Hospital, Newcastle upon Tyne
}

The constant anatomical relation of the aortic arch and the pulmonary artery bifurcation permits echocardiographic differentiation of the two great arteries when viewed from the suprasternal notch. When this technique was employed with contrast echocardiography in 12 infants, 6 of whom had ventriculo-arterial discordance (transposition of the great arteries), it was possible to establish with certainty in all but 1 case which great artery was most directly connected to the systemic venous return.

With a standard single probe echocardiogram, the morphology of the various cardiac structures is inferred mainly from their anteroposterior relation, though some additional subjective evidence can be obtained from their lateral relation. This approach is reliable only when the position of the heart in the chest is normal and when the connections of the various chambers and vessels are also normal. The scarcity of echocardiographic features which unequivocally differentiate intracardiac structures is a serious limitation of the technique in the diagnosis of complex congenital cardiac malformations.

In this report we describe a method for determining which great artery receives most of the systemic venous return, thus improving the accuracy with which we can detect abnormal cardiac connections such as ventriculo-arterial discordance (transposition of the great arteries). The method we describe is valid only when two great arteries and their semilunar valves have been unequivocally identified using standard parasternal echocardiography.

\section{Anatomical considerations}

In complex congenital heart disease the relation of the great arteries at their origin from the heart is extremely variable and this relation is frequently independent of their ultimate venous connections. In contrast, the anteroposterior relation of the arch of the aorta to the bifurcation of the pulmonary artery is always constant (Gray's Anatomy, 1969). This means that when the great arteries are both visualised with the echocardiographic probe in the Received for publication 23 February 1977 suprasternal notch (Goldberg, 1970) the aorta is always closer to the transducer than is the pulmonary artery. If an ultrasonic indicator (for instance 5 per cent dextrose), introduced into the peripheral systemic veins, can be detected when it reaches the great arteries (Valdes-Cruz et al., 1976) then this should allow us to identify whether the pulmonary artery or the aorta is most directly connected to the systemic venous ventricle.

\section{Technique}

An intravenous catheter (Longwell 20) is placed in a vein on the dorsum of the hand. The electrocardiographic electrodes are applied and the baby is allowed to settle with a rolled up napkin beneath its shoulders to extend the neck. When the infant is quiet, an echocardiogram is recorded with the transducer placed in the suprasternal notch. The beam is directed posteriorly and somewhat caudally until the echo-free spaces corresponding to the aorta, pulmonary artery, and left atrium are located. To be certain that the beam cuts the arch of the aorta and the bifurcation of the pulmonary artery, no portion of either semilunar valve should be seen. A $1 \mathrm{ml}$ bolus of 5 per cent dextrose is injected rapidly through the cannula during continuous recording of the echocardiogram. We used this technique retrospectively in 6 infants known to have normal ventriculoarterial connections and no significant shunts, and in 6 infants with discordant ventriculoarterial connections (transposition of the great arteries). In the group with normal ventriculoarterial connections only the pulmonary artery opacified (Fig. a). In the group with discordant 


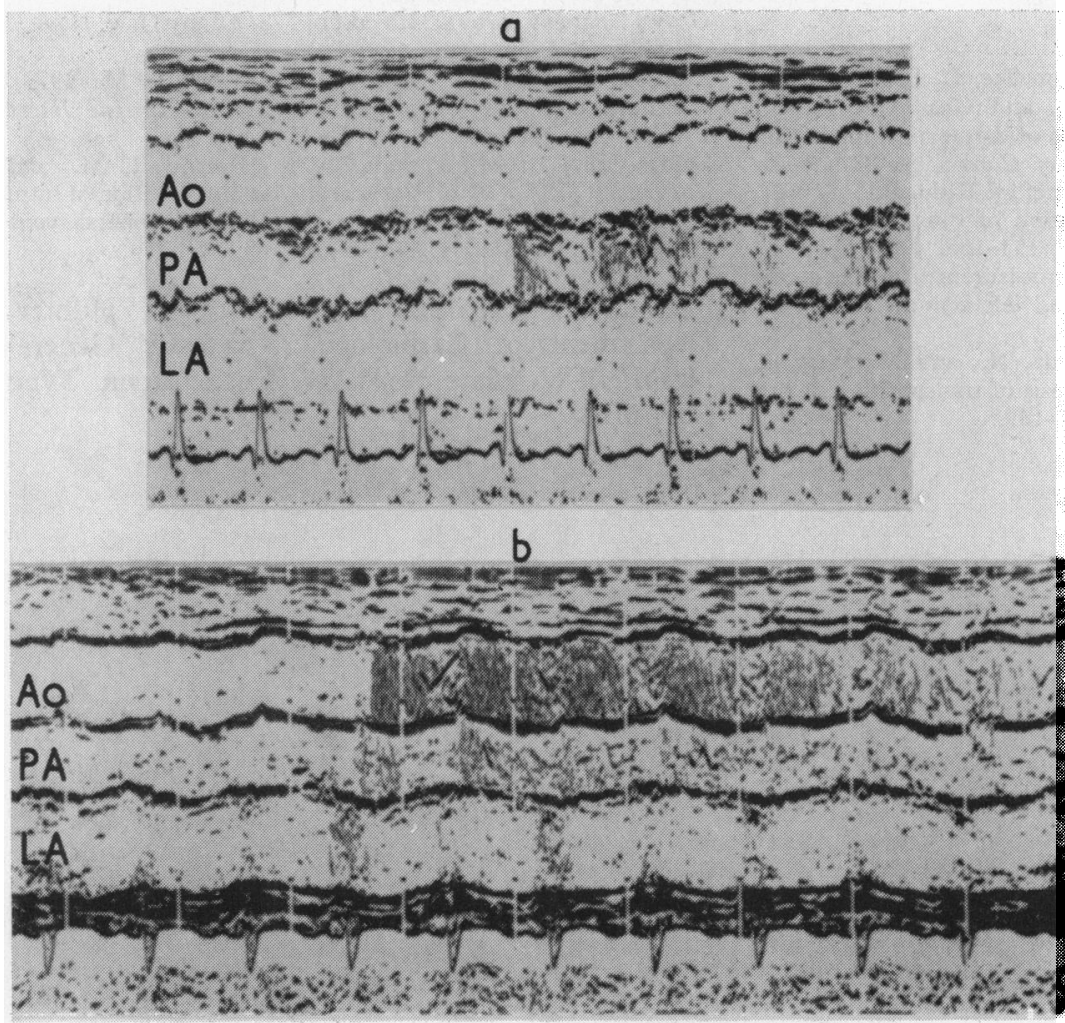

Fig. (a) This echocardiogram was recorded using the suprasternal approach in a normal infant. The aorta, pulmonary artery, and left atrium are seen. Only the pulmonary artery is opacified after dextrose injection.

(b) This echocardiogram is from an infant with transposition of the great arteries after balloon atrial septostomy. It is recorded from the suprasternal approach and shows opacification of both great arteries and the left atrium. The aorta opacifies with greater intensity than the pulmonary artery indicating that it is directly connected to the systemic venous return.

connections (transposition of the great arteries) both great arteries opacified because of the obligatory bidirectional shunting (Fig. b). In 5 of this group the aortic opacification was more intense than that of the pulmonary artery, correctly identifying the aorta as the great artery most directly connected to the systemic venous return. In the sixth case with discordant ventriculo-arterial connections, a large ventricular septal defect, and free intercirculatory communication, the great arteries were opacified with equal intensity, so that no judgement about venous connections could be made. With this technique we have subsequently correctly predicted the diagnosis of discordant ventriculo-arterial connections (transposition of the great arteries) in one neonate before cardiac catheterisation.

\section{Comment}

A major practical problem in neonatal cardiology is deciding which infants need emergency cardiac catheterisation. Standard single probe echocardio- graphy can give valuable information about complex cardiac lesions such as the hypoplastic left heart syndrome (Godman et al., 1974), or primitive ventricle (Mortera et al., 1977). But previously reported echocardiographic criteria for the diagnosis of transposition of the great arteries (Gramiak et al., 1973; Dillon et al., 1973) have not proved absolutely reliable in this age-group. The combination of the suprasternal approach with contrast echocardiography increases our diagnostic precision by identifying patients with discordant ventriculoarterial connections.

When both great arteries opacify with equal intensity it is impossible to infer the ventriculoarterial connections. In this situation the decision to catheterise or not must be made using other criteria. However, the method should identify, in the majority of cases, those infants with discordant ventriculo-arterial connections (transposition of the great arteries) with poor intercirculatory mixing, in fact those most in need of an urgent atrial septostomy. 


\section{References}

Dillon, J. C., Feigenbaum, H., Konecke, L. L., Keutel, J., Hurwitz, R. A., Davis, R. H., and Chang, S. (1973). Echocardiographic manifestations of d-transposition of the great vessels. American fournal of Cardiology, 32, 74-78.

Godman, M. J., Tham, P., and Langford Kidd, B. S. (1974). Echocardiography in the evaluation of cyanotic newborn infant. British Heart fournal, 36, 154-166.

Goldberg, B. B. (1970). Ultrasonic measurement of the aortic arch, right pulmonary artery, and left atrium. Radiology, 101, 383-390.

Gramiak, R., Chung, K. J., Nanda, N., and Manning, J. (1973). Echocardiographic diagnosis of transposition of the great vessels. Radiology, 106, 187-189.
Gray's Anatomy (1969). 34th ed., p. 778. Ed. by D. V. Davies. Longman, Edinburgh.

Mortera, C., Hunter, S., Terry, G., and Tynan, M. (1977). Echocardiography of primitive ventricle. British Heart fournal. In the press.

Valdes-Cruz, L. M., Pieroni, D. R., Roland, J. M., and Varghese, P. J. (1976). Echocardiographic detection of intra cardiac right to left shunts following peripheral vein injections. Circulation, 54, 558-562.

Requests for reprints to Dr. Stewart Hunter, Department of Cardiology, Newcastle General Hospital, Westgate Road, Newcastle upon Tyne NE4 6BE. 